\title{
Variational Homotopy Perturbation Method for Solving Higher Dimensional Initial Boundary Value Problems
}

\author{
Muhammad Aslam Noor and Syed Tauseef Mohyud-Din \\ Department of Mathematics, COMSATS Institute of Information Technology, Islamabad 44000, Pakistan
}

Correspondence should be addressed to Muhammad Aslam Noor, noormaslam@hotmail.com

Received 14 January 2008; Revised 28 March 2008; Accepted 28 May 2008

Recommended by David Chelidze

We suggest and analyze a technique by combining the variational iteration method and the homotopy perturbation method. This method is called the variational homotopy perturbation method (VHPM). We use this method for solving higher dimensional initial boundary value problems with variable coefficients. The developed algorithm is quite efficient and is practically well suited for use in these problems. The proposed scheme finds the solution without any discritization, transformation, or restrictive assumptions and avoids the round-off errors. Several examples are given to check the reliability and efficiency of the proposed technique.

Copyright (C) 2008 M. A. Noor and S. T. Mohyud-Din. This is an open access article distributed under the Creative Commons Attribution License, which permits unrestricted use, distribution, and reproduction in any medium, provided the original work is properly cited.

\section{Introduction}

The numerical and analytical solutions of higher dimensional initial boundary value problems of variable coefficients, linear and nonlinear, are of considerable significance for applied sciences. Examples of linear models are Euler-Darboux equation [1], Lambropoubs' equation [2] and Tricomi equation [3] given by

$$
\begin{gathered}
(x-y) u_{x y}+\left(\alpha u_{x}-\beta u_{y}\right)=0, \\
u_{x y}+a x u_{x}+b y u_{y}+c x y u+u_{t}=0, \\
u_{y y}=y u_{x x}
\end{gathered}
$$

respectively. Examples of nonlinear models are introduced in Kdv equation [4-7] of variable coefficients and Clairaut's equation [5] given by

$$
u_{t}+\alpha t^{n} u u_{x}+\beta t^{m} u_{x x x}=0, \quad u=x u_{x}+y u_{y}+f\left(u_{x}, u_{y}\right),
$$


respectively; see [1-7]. Several numerical and analytical techniques including the spectral methods, characteristics method, and Adomian's decomposition method have been developed for solving these problems; see [1-7] and the references therein. For implementation of the Adomian decomposition method, one has to find the so-called the Adomian polynomial, which is itself a difficult problem. To overcome these difficulties and drawbacks, He [8-18] developed variational iteration method for solving linear and nonlinear problems, which arise in various branches of pure and applied sciences. It is worth mentioning that the origin of variational iteration method can be traced back to Inokuti et al. [19]. It has been shown that the variational iteration method is user friendly. Furthermore, He [8-14] also introduced the homotopy perturbation method, which is developed by combining the standard homotopy and perturbation method. In these methods the solution is given in an infinite series usually converging to an accurate solution, see [8-31]. We would like to mention that Noor [32] used the homotopy perturbation method for suggesting a number of iterative methods solving nonlinear equations of the type $f(x)=0$. This is another application of the homotopy perturbation method.

Motivated and inspired by the on-going research in these areas, we consider a new method, which is called the variational homotopy perturbation method (VHPM). This method is suggested by combining the variational iteration technique and the homotopy perturbation method. The suggested VHPM provides the solution in a rapid convergent series which may lead the solution in a closed form and is in full agreement with [7], where similar problems were solved by using the decomposition method. The fact that the proposed technique solves nonlinear problems without using the so-called Adomian's polynomials is a clear advantage of this algorithm over the decomposition method. In this algorithm, the correct functional is developed $[8,15-19,21-25]$ and the Lagrange multipliers are calculated optimally via variational theory. Finally, the homotopy perturbation is implemented on the correct functional and the comparison of like powers of $p$ gives solutions of various orders. The developed algorithm takes full advantage of variational iteration and the homotopy perturbation methods. It is worth mentioning that the VHPM is applied without any discretization, restrictive assumption, or transformation and is free from round-off errors. Unlike the method of separation of variables that require initial and boundary conditions, the VHPM provides an analytical solution by using the initial conditions only. The boundary conditions can be used only to justify the obtained result. The proposed method work efficiently and the results so far are very encouraging and reliable. We would like to emphasize that the VHPM may be considered as an important and significant refinement of the previously developed techniques and can be viewed as an alternative to the recently developed methods such as Adomian's decomposition, variational iterations, and homotopy perturbation methods. Several examples are given to verify the reliability and efficiency of the variational homotopy perturbation method (VHPM).

\section{Variational iteration method}

To illustrate the basic concept of the technique, we consider the following general differential equation:

$$
L u+N u=g(x)
$$


where $L$ is a linear operator, $N$ a nonlinear operator, and $g(x)$ the forcing term. According to variational iteration method $[8,15-19,21-25]$, we can construct a correct functional as follows:

$$
u_{n+1}(x)=u_{n}(x)+\int_{0}^{x} \lambda\left(L u_{n}(s)+N \tilde{u}_{n}(s)-g(s)\right) d s,
$$

where $\lambda$ is a Lagrange multiplier $[8,15-19]$, which can be identified optimally via a variational iteration method. The subscripts $n$ denote the $n$th approximation, $\tilde{u}_{n}$ is considered as a restricted variation. That is, $\delta \tilde{u}_{n}=0 ;(2.2)$ is called a correct functional. The solution of the linear problems can be solved in a single iteration step due to the exact identification of the Lagrange multiplier. The principles of the variational iteration method and its applicability for various kinds of differential equations are given in [8,15-19]. In this method, it is required first to determine the Lagrange multiplier $\lambda$ optimally. The successive approximation $u_{n+1}, n \geq 0$ of the solution $u$ will be readily obtained upon using the determined Lagrange multiplier and any selective function $u_{0}$, consequently, the solution is given by $u=\lim _{n \rightarrow \infty} u_{n}$.

\section{Homotopy perturbation method}

To explain the homotopy perturbation method, we consider a general equation of the type,

$$
L(u)=0,
$$

where $L$ is any integral or differential operator. We define a convex homotopy $H(u, p)$ by

$$
H(u, p)=(1-p) F(u)+p L(u),
$$

where $F(u)$ is a functional operator with known solutions $v_{0}$, which can be obtained easily. It is clear that, for

$$
H(u, p)=0,
$$

we have

$$
H(u, 0)=F(u), \quad H(u, 1)=L(u) .
$$

This shows that $H(u, p)$ continuously traces an implicitly defined curve from a starting point $H\left(v_{0}, 0\right)$ to a solution function $H(f, 1)$. The embedding parameter monotonically increases from zero to unit as the trivial problem $F(u)=0$ is continuously deforms the original problem $L(u)=0$. The embedding parameter $p \in(0,1]$ can be considered as an expanding parameter [8-14, 26-31]. The homotopy perturbation method uses the homotopy parameter $p$ as an expanding parameter [8-14] to obtain

$$
u=\sum_{i=0}^{\infty} p^{i} u_{i}=u_{0}+p u_{1}+p^{2} u_{2}+p^{3} u_{3}+\cdots
$$

If $p \rightarrow 1$, then (3.5) corresponds to (3.2) and becomes the approximate solution of the form

$$
f=\lim _{p \rightarrow 1} u=\sum_{i=0}^{\infty} u_{i}
$$

It is well known that series (3.5) is convergent for most of the cases and also the rate of convergence is dependent on $L(u)$; see [8-14]. We assume that (3.6) has a unique solution. The comparisons of like powers of $p$ give solutions of various orders. 


\section{Variational homotopy perturbation method (VHPM)}

To convey the basic idea of the variational homotopy perturbation method, we consider the following general differential equation:

$$
L u+N u=g(x)
$$

where $L$ is a linear operator, $N$ a nonlinear operator, and $g(x)$ the forcing term. According to variational iteration method $[8,15-19]$, we can construct a correct functional as follows:

$$
u_{n+1}(x)=u_{n}(x)+\int_{0}^{x} \lambda(\xi)\left(L u_{n}(\xi)+N \tilde{u}_{n}(\xi)-g(\xi)\right) d \xi,
$$

where $\lambda$ is a Lagrange multiplier $[8,15-19]$, which can be identified optimally via variational iteration method. The subscripts $n$ denote the $n$th approximation, $\tilde{u}_{n}$ is considered as a restricted variation. That is, $\delta \widetilde{u}_{n}=0 ;(4.2)$ is called as a correct functional. Now, we apply the homotopy perturbation method,

$$
\sum_{n=0}^{\infty} p^{(n)} u_{n}=u_{0}(x)+p \int_{0}^{x} \lambda(\xi)\left(\sum_{n=0}^{\infty} p^{(n)} L\left(u_{n}\right)+\sum_{n=0}^{\infty} p^{(n)} N\left(\tilde{u}_{n}\right)\right) d \xi-\int_{0}^{x} \lambda(\xi) g(\xi) d \xi,
$$

which is the variational homotopy perturbation method and is formulated by the coupling of variational iteration method and Adomian's polynomials. A comparison of like powers of $p$ gives solutions of various orders.

\section{Numerical applications}

In this section, we apply the VHPM developed in Section 4 for solving higher dimensional initial boundary value problems with variable coefficient. We develop the correct functional and calculate the Lagrange multipliers optimally via variational theory. The homotopy perturbation method is implemented on the correct functional and finally, the comparison of like powers of $p$ gives solutions of various orders. Numerical results reveal that the VHPM is easy to implement and reduces the computational work to a tangible level while still maintaining a very higher level of accuracy. For the sake of comparison, we take the same examples as used in $[7,20]$.

Example 5.1. Consider the two-dimensional initial boundary value problem:

$$
u_{t t}=\frac{1}{2} y^{2} u_{x x}+\frac{1}{2} x^{2} u_{y y}, \quad 0<x, y<1, t>0,
$$

with boundary conditions

$$
\begin{array}{ll}
u(0, y, t)=y^{2} e^{-t}, & u(1, y, t)=\left(1+y^{2}\right) e^{-t}, \\
u(x, 0, t)=y^{2} e^{-t}, & u(x, 1, t)=\left(1+x^{2}\right) e^{-t},
\end{array}
$$

and the initial conditions

$$
u(x, y, 0)=x^{2}+y^{2}, \quad u_{t}(x, y, 0)=-\left(x^{2}+y^{2}\right) .
$$


The correct functional is given as

$$
u_{n+1}(x, y, z, t)=\left(x^{2}+y^{2}\right)-\left(x^{2}+y^{2}\right) t+\int_{0}^{t} \lambda(\xi)\left(\frac{\partial^{2} u_{n}}{\partial t^{2}}-\frac{1}{2}\left(y^{2}\left(\tilde{u}_{n}\right)_{x x}+x^{2}\left(\tilde{u}_{n}\right)_{y y}\right)\right) d \xi
$$

where $\tilde{u}_{n}$ is considered as a restricted variation. Making the above functional stationary, the Lagrange multiplier can be determined as $\lambda=\xi-t$, which yields the following iteration formula:

$$
u_{n+1}(x, y, z, t)=\left(x^{2}+y^{2}\right)-\left(x^{2}+y^{2}\right) t+\int_{0}^{t}(\xi-t)\left(\frac{\partial^{2} u_{n}}{\partial t^{2}}-\frac{1}{2}\left(y^{2}\left(u_{n}\right)_{x x}+x^{2}\left(u_{n}\right)_{y y}\right)\right) d \xi
$$

Applying the variational homotopy perturbation method, we have

$$
\begin{aligned}
u_{0}+p u_{1}+p^{2} u_{2}+\cdots= & \left(x^{2}+y^{2}\right)-\left(x^{2}+y^{2}\right) t+p \int_{0}^{t}(\xi-t)\left(\frac{\partial^{2} u_{0}}{\partial t^{2}}+p \frac{\partial^{2} u_{1}}{\partial t^{2}}+p^{2} \frac{\partial^{2} u_{2}}{\partial t^{2}}+\cdots\right) d \xi \\
-\frac{1}{2} p \int_{0}^{t}(\xi-t)( & \left(y^{2}\left(\frac{\partial^{2} \widetilde{u}_{0}}{\partial x^{2}}+p \frac{\partial^{2} \widetilde{u}_{1}}{\partial x^{2}}+p^{2} \frac{\partial^{2} \widetilde{u}_{2}}{\partial x^{2}}+\cdots\right)\right) \\
& \left.+\left(x^{2}\left(\frac{\partial^{2} \widetilde{u}_{0}}{\partial y^{2}}+p \frac{\partial^{2} \widetilde{u}_{1}}{\partial y^{2}}+p^{2} \frac{\partial^{2} \widetilde{u}_{2}}{\partial y^{2}}+\cdots\right)\right)\right) d \xi .
\end{aligned}
$$

Comparing the coefficient of like powers of $p$, we have

$$
\begin{aligned}
& p^{(0)}: u_{0}(x, y, t)=\left(x^{2}+y^{2}\right)-\left(x^{2}+y^{2}\right) t \\
& p^{(1)}: u_{1}(x, y, t)=\left(x^{2}+y^{2}\right) \frac{t^{2}}{2 !}-\left(x^{2}+y^{2}\right) \frac{t^{3}}{3 !^{\prime}} \\
& p^{(2)}: u_{2}(x, y, t)=\left(x^{2}+y^{2}\right) \frac{t^{4}}{4 !}-\left(x^{2}+y^{2}\right) \frac{t^{5}}{5 !^{\prime}} \\
& p^{(3)}: u_{3}(x, y, t)=\left(x^{2}+y^{2}\right) \frac{t^{5}}{5 !}-\left(x^{2}+y^{2}\right) \frac{t^{7}}{7 !^{\prime}} \\
& p^{(4)}: u_{4}(x, y, t)=\left(x^{2}+y^{2}\right) \frac{t^{8}}{8 !}-\left(x^{2}+y^{2}\right) \frac{t^{9}}{9 !^{\prime}} \\
& p^{(5)}: u_{5}(x, y, t)=\left(x^{2}+y^{2}\right) \frac{t^{10}}{10 !}-\left(x^{2}+y^{2}\right) \frac{t^{11}}{11 !^{\prime}}
\end{aligned}
$$

The series solution is given by

$$
u(x, y, t)=\left(x^{2}+y^{2}\right)\left(1-t+\frac{t^{2}}{2 !}-\frac{t^{3}}{3 !}+\frac{t^{4}}{4 !}-\frac{t^{5}}{5 !}+\frac{t^{6}}{6 !}-\frac{t^{7}}{7 !}+\frac{t^{8}}{8 !}-\cdots\right)
$$

and in a closed form by $u(x, y, t)=\left(x^{2}+y^{2}\right) e^{-t}$, which is in full agreement with [7]. 
Example 5.2. Consider the three-dimensional initial boundary value problem

$$
u_{t t}=\frac{1}{45} x^{2} u_{x x}+\frac{1}{45} y^{2} u_{y y}+\frac{1}{45} z^{2} u_{z z}-u, \quad 0<x, y<1, t<0
$$

subject to the Neumann boundary conditions

$$
\begin{gathered}
u_{x}(0, y, z, t)=0, \quad u_{x}(1, y, z, t)=6 y^{6} z^{6} \sinh t, \quad u_{y}(x, 0, z, t)=0, \\
u_{y}(x, 1, z, t)=6 x^{6} z^{6} \sinh t, \quad u_{z}(x, y, 0, t)=0, \quad u_{z}(x, y, 1, t)=6 x^{6} y^{6} \sinh t
\end{gathered}
$$

and the initial conditions

$$
u(x, y, z, 0)=0, \quad u_{t}(x, y, z, 0)=x^{6} y^{6} z^{6}
$$

The correct functional is given by

$$
u_{n+1}(x, y, z, t)=\left(x^{6} y^{6} z^{6}\right) t+\int_{0}^{t} \lambda(\xi)\left(\frac{\partial^{2} u_{n}}{\partial t^{2}}-\frac{1}{45}\left(x^{2}\left(\tilde{u}_{n}\right)_{x x}+y^{2}\left(\tilde{u}_{n}\right)_{y y}+z^{2}\left(\tilde{u}_{n}\right)_{z z}\right)+\tilde{u}_{n}\right) d \xi,
$$

where $\tilde{u}_{n}$ is considered as a restricted variation. Making the above functional stationary, the Lagrange multiplier can be determined as $\lambda=\xi-t$, which yields the following iteration formula:

$$
u_{n+1}(x, y, z, t)=\left(x^{6} y^{6} z^{6}\right) t+\int_{0}^{t}(\xi-t)\left(\frac{\partial^{2} u_{n}}{\partial t^{2}}-\frac{1}{45}\left(x^{2}\left(u_{n}\right)_{x x}+y^{2}\left(u_{n}\right)_{y y}+z^{2}\left(u_{n}\right)_{z z}\right)+u_{n}\right) d \xi
$$

Applying the variational homotopy perturbation method,

$$
\begin{aligned}
u_{0}+p u_{1}+p^{2} u_{2}+\cdots= & \left(x^{2}+y^{2}\right)-\left(x^{2}+y^{2}\right) t+p \int_{0}^{t}(\xi-t)\left(\frac{\partial^{2} u_{0}}{\partial t^{2}}+p \frac{\partial^{2} u_{1}}{\partial t^{2}}+p^{2} \frac{\partial^{2} u_{2}}{\partial t^{2}}+\cdots\right) d \xi \\
& -\frac{1}{45} p \int_{0}^{t}(\xi-t)\left(\left(x^{2}\left(\frac{\partial^{2} \widetilde{u}_{0}}{\partial x^{2}}+p \frac{\partial^{2} \widetilde{u}_{1}}{\partial x^{2}}+p^{2} \frac{\partial^{2} \widetilde{u}_{2}}{\partial x^{2}}+\cdots\right)\right)\right. \\
& \left.+\left(y^{2}\left(\frac{\partial^{2} \widetilde{u}_{0}}{\partial y^{2}}+p \frac{\partial^{2} \widetilde{u}_{1}}{\partial y^{2}}+p^{2} \frac{\partial^{2} \widetilde{u}_{2}}{\partial y^{2}}+\cdots\right)\right)\right) d \xi \\
& -\frac{1}{45} p \int_{0}^{t}(\xi-t) z^{2}\left(\frac{\partial^{2} \widetilde{u}_{0}}{\partial z^{2}}+p \frac{\partial^{2} \widetilde{u}_{1}}{\partial z^{2}}+p^{2} \frac{\partial^{2} \widetilde{u}_{2}}{\partial z^{2}}+\cdots\right) d \xi \\
& +p \int_{0}^{t}(\xi-t)\left(u_{0}+p u_{1}+p^{2} u_{2}+\cdots\right) d \xi .
\end{aligned}
$$


Comparing the coefficient of like powers of $p$, we have

$$
\begin{aligned}
& p^{(0)}: u_{0}(x, y, z, t)=x^{6} y^{6} z^{6} t, \\
& p^{(1)}: u_{1}(x, y, z, t)=x^{6} y^{6} z^{6} \frac{t^{3}}{3 !}, \\
& p^{(2)}: u_{2}(x, y, z, t)=x^{6} y^{6} z^{6} \frac{t^{5}}{5 !}, \\
& p^{(3)}: u_{3}(x, y, z, t)=x^{6} y^{6} z^{6} \frac{t^{7}}{7 !}, \\
& p^{(4)}: u_{4}(x, y, z, t)=x^{6} y^{6} z^{6} \frac{t^{9}}{9 !}, \\
& \vdots
\end{aligned}
$$

The series solution is given by

$$
u(x, y, z, t)=x^{6} y^{6} z^{6}\left(t+\frac{t^{3}}{3 !}+\frac{t^{5}}{5 !}+\frac{t^{7}}{7 !}+\frac{t^{9}}{9 !}+\cdots\right)=x^{6} y^{6} z^{6} \sinh t
$$

which is in full agreement with [7].

Example 5.3. Consider the two-dimensional nonlinear inhomogeneous initial boundary value problem

$$
u_{t t}=2 x^{2}+2 y^{2}+\frac{15}{2}\left(x u_{x x}^{2}+y u_{y y}^{2}\right), \quad 0<x, y<1, t>0
$$

with boundary conditions

$$
\begin{array}{ll}
u(0, y, t)=y^{2} t^{2}+y t^{6}, & u(1, y, t)=\left(1+y^{2}\right) t^{2}+(1+y) t^{6}, \\
u(x, 0, t)=x^{2} t^{2}+x t^{6}, & u(x, 1, t)=\left(1+x^{2}\right) t^{2}+(1+x) t^{6}
\end{array}
$$

and the initial conditions

$$
u(x, y, 0)=0, \quad u_{t}(x, y, 0)=0,
$$

The correct functional is given as

$$
u_{n+1}(x, y, z, t)=\int_{0}^{t} \lambda(\xi)\left(\frac{\partial^{2} u_{n}}{\partial t^{2}}-\frac{15}{2}\left(x\left(\tilde{u}_{n}^{2}\right)_{x x}+y^{2}\left(\tilde{u}_{n}^{2}\right)_{y y}\right)-2\left(x^{2}+y^{2}\right)\right) d \xi
$$


where $\tilde{u}_{n}$ is considered as a restricted variation. Making the above functional stationary, the Lagrange multiplier can be determined as $\lambda=\xi-t$, which yields the following iteration formula

$$
u_{n+1}(x, y, z, t)=\int_{0}^{t}(\xi-t)\left(\frac{\partial^{2} u_{n}}{\partial t^{2}}-\frac{15}{2}\left(x\left(\tilde{u}_{n}^{2}\right)_{x x}+y^{2}\left(\widetilde{u}_{n}^{2}\right)_{y y}\right)-2\left(x^{2}+y^{2}\right)\right) d \xi .
$$

Applying the variational homotopy perturbation method, we have

$$
\begin{aligned}
u_{0}+p u_{1}+p^{2} u_{2}+\cdots= & p \int_{0}^{t}(\xi-t)\left(\frac{\partial^{2} u_{0}}{\partial t^{2}}+p \frac{\partial^{2} u_{1}}{\partial t^{2}}+p^{2} \frac{\partial^{2} u_{2}}{\partial t^{2}}+\cdots\right) d \xi \\
-\frac{15}{2} p(\xi-t)( & x\left(\frac{\partial^{2} \tilde{u}_{0}}{\partial x^{2}}+p \frac{\partial^{2} \widetilde{u}_{1}}{\partial x^{2}}+p^{2} \frac{\partial^{2} \widetilde{u}_{2}}{\partial x^{2}}+\cdots\right)_{x x}^{2} \\
& \left.+\int_{0}^{t} y\left(\frac{\partial^{2} \widetilde{u}_{0}}{\partial y^{2}}+p \frac{\partial^{2} \tilde{u}_{1}}{\partial y^{2}}+p^{2} \frac{\partial^{2} \tilde{u}_{2}}{\partial y^{2}}+\cdots\right)_{y y}^{2}-2\left(x^{2}+y^{2}\right)\right) d \xi .
\end{aligned}
$$

Comparing the coefficient of like powers of $p$, we have

$$
\begin{aligned}
& p^{(0)}: u_{0}(x, y, t)=0 \\
& p^{(1)}: u_{1}(x, y, t)=\left(x^{2}+y^{2}\right) t^{2}, \\
& p^{(2)}: u_{2}(x, y, t)=(x+y) t^{6} \\
& p^{(3)}: u_{3}(x, y, t)=0 \\
& \vdots
\end{aligned}
$$

The solution is obtained as $u(x, y, t)=\left(x^{2}+y^{2}\right) t^{2}+(x+y) t^{6}$, which is in full agreement with [7].

Example 5.4. Consider the three-dimensional nonlinear initial boundary value problem

$$
u_{t t}=\left(2-t^{2}\right)+u-\left(e^{-x} u_{x x}^{2}+e^{-y} u_{y y}^{2}+e^{-z} u_{z z}^{2}\right), \quad 0<x, y<1, t<0
$$

subject to the Neumann boundary conditions

$$
\begin{array}{ll}
u_{x}(0, y, z, t)=1, & u_{x}(1, y, z, t)=e, \\
u_{y}(x, 0, z, t)=0, & u_{y}(x, 1, z, t)=e, \\
u_{z}(x, y, 0, t)=1, & u_{z}(x, y, 1, t)=e,
\end{array}
$$

and the initial conditions

$$
u(x, y, z, 0)=e^{x}+e^{y}+e^{z}, \quad u_{t}(x, y, z, 0)=0 .
$$


M. A. Noor and S. T. Mohyud-Din

The correct functional is given as

$$
\begin{aligned}
& u_{n+1}(x, y, z, t) \\
& =\left(e^{x}+e^{y}+e^{z}\right)+\int_{0}^{t} \lambda(\xi)\left(\frac{\partial^{2} u_{n}}{\partial t^{2}}+\left(e^{-x}\left(\widetilde{u}_{n}\right)_{x x}^{2}+e^{-y}\left(\widetilde{u}_{n}\right)_{y y}^{2}+e^{-z}\left(\widetilde{u}_{n}\right)_{z z}^{2}\right)-\tilde{u}_{n}\right) d \xi-\int_{0}^{t} \lambda(\xi)\left(2-t^{2}\right) d \xi
\end{aligned}
$$

where $\tilde{u}_{n}$ is considered as a restricted variation. Making the above functional stationary, the Lagrange multiplier can be determined as $\lambda=\xi-t$, which yields the following iteration formula:

$$
\begin{aligned}
& u_{n+1}(x, y, z, t) \\
& =\left(e^{x}+e^{y}+e^{z}\right)+\int_{0}^{t}(\xi-t)\left(\frac{\partial^{2} u_{n}}{\partial t^{2}}+\left(e^{-x}\left(\tilde{u}_{n}\right)_{x x}^{2}+e^{-y}\left(\tilde{u}_{n}\right)_{y y}^{2}+e^{-z}\left(\tilde{u}_{n}\right)_{z z}^{2}\right)-\tilde{u}_{n}\right) d \xi-\int_{0}^{t} \lambda(\xi)\left(2-t^{2}\right) d \xi,
\end{aligned}
$$

Applying the variational homotopy perturbation method, we have

$$
\begin{aligned}
& u_{0}+p u_{1}+\cdots=\left(e^{x}+e^{y}+e^{z}\right)+p \int_{0}^{t}(\xi-t)\left(\left(\frac{\partial^{2} u_{0}}{\partial t^{2}}+p \frac{\partial^{2} u_{1}}{\partial t^{2}}+p^{2} \frac{\partial^{2} u_{2}}{\partial t^{2}}+\cdots\right)\right. \\
& \left.+e^{-x}\left(\frac{\partial^{2} \tilde{u}_{0}}{\partial x^{2}}+p \frac{\partial^{2} \tilde{u}_{1}}{\partial x^{2}}+p^{2} \frac{\partial^{2} \tilde{u}_{2}}{\partial x^{2}}+\cdots\right)_{x x}^{2}\right) d \xi \\
& +p \int_{0}^{t}(\xi-t)\left(e^{-y}\left(\frac{\partial^{2} \tilde{u}_{0}}{\partial y^{2}}+p \frac{\partial^{2} \tilde{u}_{1}}{\partial y^{2}}+p^{2} \frac{\partial^{2} \tilde{u}_{2}}{\partial y^{2}}+\cdots\right)_{x x}^{2}\right. \\
& \left.+\int_{0}^{t} e^{-z}\left(\frac{\partial^{2} \tilde{u}_{0}}{\partial z^{2}}+p \frac{\partial^{2} \tilde{u}_{1}}{\partial z^{2}}+p^{2} \frac{\partial^{2} \tilde{u}_{2}}{\partial z^{2}}+\cdots\right)_{y y}^{2}\right) d \xi \\
& -p \int_{0}^{t}(\xi-t)\left(\left(u_{0}+p u_{1}+p^{2} u_{2}+\cdots\right)+\left(2-t^{2}\right)\right) d \xi .
\end{aligned}
$$

Comparing the coefficient of like powers of $p$, we have

$$
\begin{aligned}
& p^{(0)}: u_{0}(x, y, z, t)=\left(e^{x}+e^{y}+e^{z}\right)+t^{2}-\frac{t^{4}}{12} \\
& p^{(1)}: u_{1}(x, y, z, t)=\frac{t^{4}}{12}-\frac{t^{6}}{360} \\
& p^{(2)}: u_{2}(x, y, z, t)=\frac{t^{6}}{360}-\frac{t^{8}}{20160}
\end{aligned}
$$

The solution is obtained as $u(x, y, z, t)=\left(e^{x}+e^{y}+e^{z}\right)+t^{2}$, which is in full agreement with [7]. 
Remark 5.5. We would like to point out that Noor [32] used the homotopy perturbation method for suggesting some iterative-type methods for solving nonlinear equations $f(x)=0$ coupled with system of equations. Also it has been shown [32] that the homotopy perturbation method and Adomian decomposition method are equivalent. This application of the homotopy method is quite different in nature. It is an interesting problem to consider such type of applications of the variational homotopy method in solving nonlinear equations.

\section{Conclusions}

In this paper, we develop the variational homotopy perturbation method (VHPM) for solving nonlinear problems. We used the variational homotopy perturbation method for solving the higher dimensional initial boundary value problems with variable coefficient. The proposed method is successfully implemented by using the initial conditions only. There are two important points to make here. First, unlike the implicit and explicit finite difference methods, the solution here is given in a closed form and by using the initial conditions only. Second, the VHPM avoids the cumbersome of the computational methods while still maintaining the higher level of accuracy. The fact that the variational homotopy perturbation method solves nonlinear problems without using the Adomian's polynomials can be considered as a clear advantage of this technique over the decomposition method. It is observed that the proposed scheme exploits full advantage of variational iteration method and the homotopy perturbation method. Finally, we conclude that the VHPM may be considered as a nice refinement in existing numerical techniques.

\section{Acknowledgments}

The authors are highly grateful to a referee for his/her constructive comments. They would like to thank Dr. S. M. Junaid Zaidi, Rector CIIT for providing excellent research environment and facilities.

\section{References}

[1] W. Miller Jr., "Symmetries of differential equations. The hypergeometric and Euler-Darboux equations," SIAM Journal on Mathematical Analysis, vol. 4, no. 2, pp. 314-328, 1973.

[2] R. Wilcox, "Closed-form solution of the differential equation $\left(\left(\partial^{2} / \partial x \partial y\right)+a x(\partial / \partial x)+b y(\partial / \partial y)+\right.$ $c x y+(\partial / \partial t)) P=0$ by normal-ordering exponential operators," Journal of Mathematical Physics, vol. 11, pp. 1235-1237, 1970.

[3] A. R. Manwell, The Tricomi Equation with Applications to the Theory of Plane Transonic Flow, vol. 35 of Research Notes in Mathematics, Pitman, London, UK, 1979.

[4] N. Nirmala, M. J. Vedan, and B. V. Baby, "A variable coefficient Korteweg-de Vries equation: similarity analysis and exact solution-II," Journal of Mathematical Physics, vol. 27, no. 11, pp. 2644-2646, 1986.

[5] S. Iyanaga and Y. Kawada, Encyclopedic Dictionary of Mathematics, MIT Press, Cambridge, Mass, USA, 1962.

[6] A. A. Soliman, "A numerical simulation and explicit solutions of KdV-Burgers' and Lax's seventhorder KdV equations," Chaos, Solitons E Fractals, vol. 29, no. 2, pp. 294-302, 2006.

[7] A.-M. Wazwaz, "The decomposition method for solving higher dimensional initial boundary value problems of variable coefficients," International Journal of Computer Mathematics, vol. 76, no. 2, pp. 159$172,2000$.

[8] J.-H. He, "Some asymptotic methods for strongly nonlinear equations," International Journal of Modern Physics B, vol. 20, no. 10, pp. 1141-1199, 2006. 
[9] J.-H. He, "Homotopy perturbation technique," Computer Methods in Applied Mechanics and Engineering, vol. 178 , no. 3-4, pp. 257-262, 1999.

[10] J.-H. He, "Homotopy perturbation method for solving boundary value problems," Physics Letters A, vol. 350, no. 1-2, pp. 87-88, 2006.

[11] J.-H. He, "Comparison of homotopy perturbation method and homotopy analysis method," Applied Mathematics and Computation, vol. 156, no. 2, pp. 527-539, 2004.

[12] J.-H. He, "Homotopy perturbation method for bifurcation of nonlinear problems," International Journal of Nonlinear Sciences and Numerical Simulation, vol. 6, no. 2, pp. 207-208, 2005.

[13] J.-H. He, "The homotopy perturbation method nonlinear oscillators with discontinuities," Applied Mathematics and Computation, vol. 151, no. 1, pp. 287-292, 2004.

[14] J.-H. He, "A coupling method of a homotopy technique and a perturbation technique for non-linear problems," International Journal of Non-Linear Mechanics, vol. 35, no. 1, pp. 37-43, 2000.

[15] J.-H. He, "Variational iteration method-a kind of non-linear analytical technique: some examples," International Journal of Non-Linear Mechanics, vol. 34, no. 4, pp. 699-708, 1999.

[16] J.-H. He, "Variational iteration method for autonomous ordinary differential systems," Applied Mathematics and Computation, vol. 114, no. 2-3, pp. 115-123, 2000.

[17] J.-H. He and X.-H. Wu, "Construction of solitary solution and compacton-like solution by variational iteration method," Chaos, Solitons \& Fractals, vol. 29, no. 1, pp. 108-113, 2006.

[18] J.-H. He and X.-H. Wu, "Variational iteration method: new development and applications," Computers $\mathcal{E}$ Mathematics with Applications, vol. 54, no. 7-8, pp. 881-894, 2007.

[19] M. Inokuti, H. Sekine, and T. Mura, "General use of the Lagrange multiplier in nonlinear mathematical physics," in Variational Method in the Mechanics of Solids, S. Nemat-Naseer, Ed., pp. 156-162, Pergamon Press, New York, NY, USA, 1978.

[20] M. A. Noor and S. T. Mohyud-Din, "Variational iteration decomposition method for solving higher dimensional initial boundary value problems with variable coefficients," preprint, 2007.

[21] S. T. Mohyud-Din, "A reliable algorithm for Blasius equation," in Proceedings of the International Conference of Mathematical Sciences (ICMS '07), pp. 616-626, Selangor, Malaysia, November 2007.

[22] M. A. Noor and S. T. Mohyud-Din, "Variational iteration technique for solving higher order boundary value problems," Applied Mathematics and Computation, vol. 189, no. 2, pp. 1929-1942, 2007.

[23] M. A. Noor and S. T. Mohyud-Din, "An efficient method for fourth-order boundary value propblems," Computers \& Mathematics with Applications, vol. 54, no. 7-8, pp. 1101-1111, 2007.

[24] M. A. Noor and S. T. Mohyud-Din, "Variational iteration technique for solving higher order boundary value problems," Applied Mathematics and Computation, vol. 189, no. 2, pp. 1929-1942, 2007.

[25] M. A. Noor and S. T. Mohyud-Din, "Variational iteration decomposition method for solving eighthorder boundary value problems," Differential Equations and Nonlinear Mechanics, vol. 2007, Article ID 19529, 16 pages, 2007.

[26] S. T. Mohyud-Din and M. A. Noor, "Homotopy perturbation method for solving fourth order boundary value problems," Mathematical Problems in Engineering, vol. 2006, Article ID 98602, 15 pages, 2006.

[27] M. A. Noor and S. T. Mohyud-Din, "Homotopy method for solving eighth order boundary value problems," Journal of Mathematical Analysis and Approximation Theory, vol. 1, no. 2, pp. 161-169, 2006.

[28] M. A. Noor and S. T. Mohyud-Din, "An efficient algorithm for solving fifth-order boundary value problems," Mathematical and Computer Modelling, vol. 45, no. 7-8, pp. 954-964, 2007.

[29] M. A. Noor and S. T. Mohyud-Din, "Homotopy perturbation method for solving sixth-order boundary value problems," Computers \& Mathematics with Applications, vol. 55, no. 12, pp. 2953-2972, 2008.

[30] M. A. Noor and S. T. Mohyud-Din, "A reliable approach for solving linear and nonlinear sixth-order boundary value problems," International Journal of Computational and Applied Mathematics, vol. 2, no. 2, pp. 163-172, 2007.

[31] M. A. Noor and S. T. Mohyud-Din, "Approximate solutions of Flieral-Petviashivili equation and its variants," International Journal of Mathematics and Computer Science, vol. 2, no. 4, pp. 345-360, 2007.

[32] M. A. Noor, "On iterative methods for nonlinear equations using homotopy perturbation technique," preprint, 2008. 


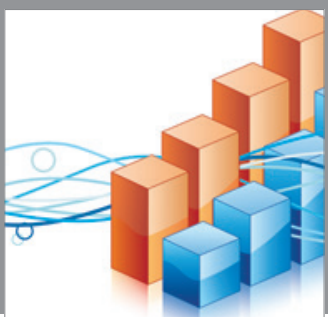

Advances in

Operations Research

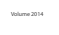

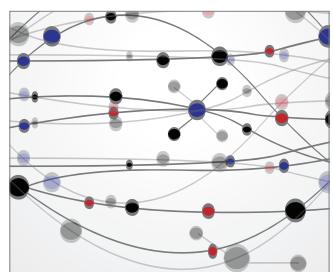

\section{The Scientific} World Journal
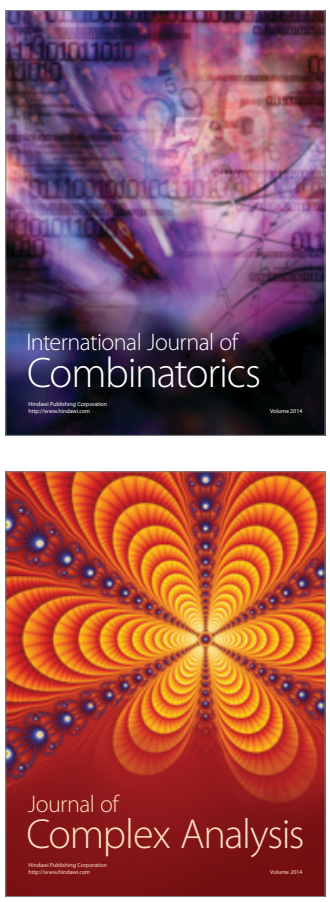

International Journal of

Mathematics and

Mathematical

Sciences
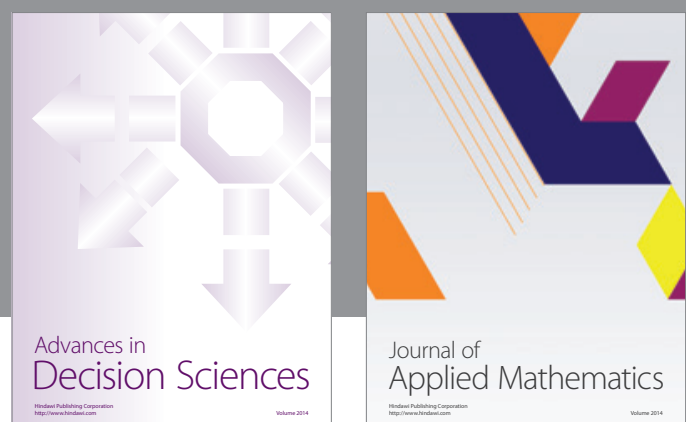

Journal of

Applied Mathematics
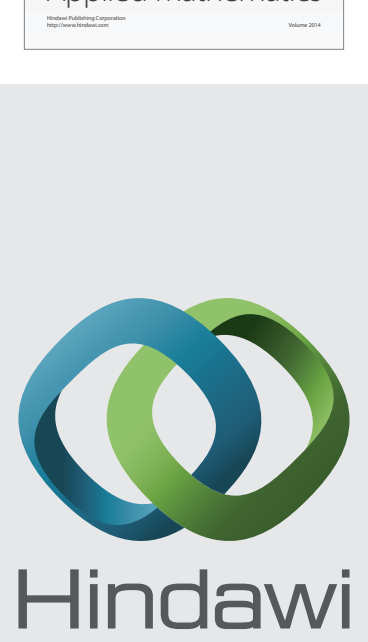

Submit your manuscripts at http://www.hindawi.com
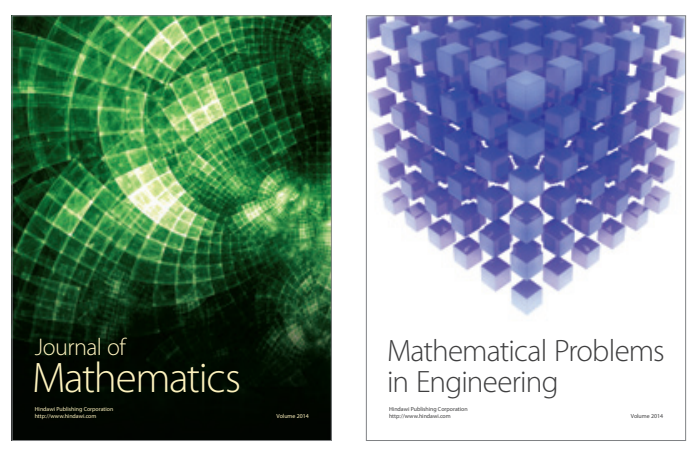

Mathematical Problems in Engineering
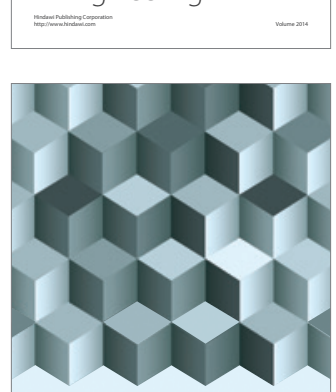

Journal of

Function Spaces
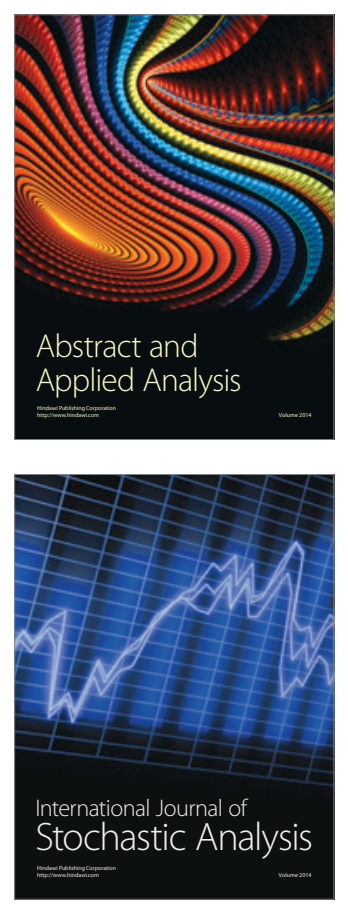

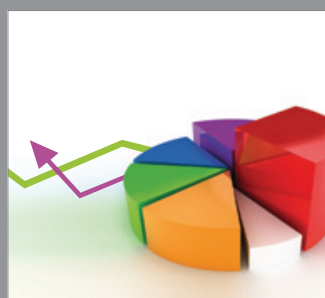

ournal of

Probability and Statistics

Promensencen
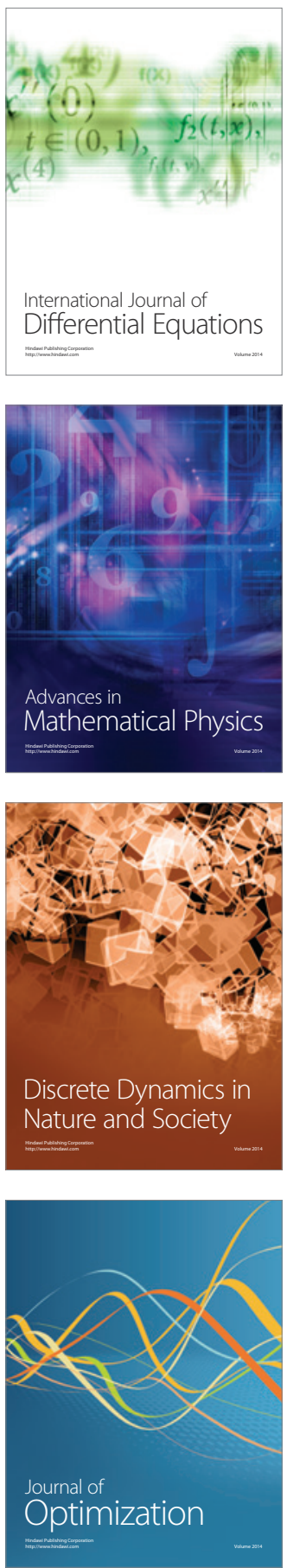